\title{
Detection and Quantification of Nuclear Morphology Changes in Apoptotic Cells by Fluorescence Microscopy and Subsequent Analysis of Visualized Fluorescent Signals
}

\author{
ROBERT MANDELKOW ${ }^{1}$, DENIS GÜMBEL ${ }^{2}$, HANNES AHREND ${ }^{1}$, ANNE KAUL ${ }^{1}$, \\ UWE ZIMMERMANN $^{1}$, MARTIN BURCHARDT ${ }^{1}$ and MATTHIAS B. STOPE ${ }^{1}$ \\ Departments of ${ }^{1}$ Urology and ${ }^{2}$ Trauma, Reconstructive Surgery and Rehabilitation Medicine, \\ University of Medicine Greifswald, Greifswald, Germany
}

\begin{abstract}
Background: Apoptosis results in specific and stage-dependent morphological alterations of the cell nucleus, including pyknosis and cell shrinking. The experimental investigation of apoptotic processes is still challenging and routinely based on the assessment of molecular events like chromatin fragmentation and caspase enzyme activity. Alternatively, the establishment of a fluorescence microscopy nuclear morphology assay would provide a simple and robust low-cost method for detection and quantification of apoptotic cascades. Materials and Methods: Model cell lines LNCaP and MDA-MB-231 were incubated in the presence of the apoptosis-inducer cycloheximide (CHX). After evaluation of apoptotic cascades by terminal deoxynucleotidyl transferase-dUTP nick-end labeling (TUNEL) assay, stained cell nuclei were analyzed regarding area, perimeter, major and minor axis, as well as brightness of nuclear fluorescence signal. Results: When compared to vehicle-treated control cells, administration of CHX led to significantly reduced cell growth and elevated rates of chromatin fragmentation of both cell lines as shown by cell counting and TUNEL assay, respectively. These apoptotic effects were accompanied by apoptosis-specific modulations of the nuclei demonstrated by diminished nuclear morphology parameters, such as area, perimeter, major and minor axis, as well as elevated levels of nuclear staining intensity. Conclusion: We present a computerized method for apoptosis detection and
\end{abstract}

This article is freely accessible online.

Correspondence to: Matthias Stope, Department of Urology, University Medicine Greifswald, Ferdinand-Sauerbruch-Straße, 17475 Greifswald, Germany. Tel: +49 38348680436, Fax: +49 38348680435, e-mail: matthias.stope@uni-greifswald.de

Key Words: Apoptosis, fluorescence microscopy, DAPI staining, nuclear morphology assay. quantification using images of fluorescent dye-stained cell nuclei. The advantages of this nuclear morphology assay include the (i) ability to routinely assess apoptosis by a fast, highly reproducible low-cost technique, (ii) applicability of an experimental approach analyzing high numbers of single nuclei and (iii) detection of apoptosis in early, as well as late, stages of the apoptotic cascade.

Highly specific inactivation of misplaced or damaged cells through induction of evolutionarily-conserved apoptotic mechanisms is crucial for maintaining tissue homeostasis. Apoptosis - the programmed cell death- is extensively correlated with cellular growth, as well as cellular stress response, and is frequently deregulated in disease pathologies. Deregulation of apoptotic cascades are associated with a wide range of diseases, particularly, involved in cancer. Thus, induction of apoptosis is the primary goal for cancer prevention, as various anticancer drugs exert their anti-oncogenic efficacy by inducing apoptosis (1-3).

Apoptosis consists of a series of highly organized and fine-tuned processes accompanied with structural changes in cellular and sub-cellular architecture firstly described in the 1970's by Kerr and co-workers (4). Besides changes in energy-dependent molecular pathways, induction of apoptosis also results in characteristic and stage-dependent morphological alterations, including chromatin condensation (pyknosis), cell shrinking, nuclear deformation, formation of apoptotic bodies and subsequent degradation of the apoptotic bodies by adjacent cells (5).

Experimental measurement of apoptosis, however, is still challenging. Routinely, apoptosis is demonstrated by assessment of apoptosis-specific molecular events like nuclear DNA fragmentation (terminal deoxynucleotidyl transferase-UTP nick-end labeling (TUNEL) assay, comet assay), caspase enzyme activity (caspase assay) and microscopy analysis of the phosphatidylserine translocation to the outer face of the cytoplasmic membrane by annexin 
V-coupled fluorescent dyes (annexin assay). Moreover, apoptosis has often been determined by Western blotting and quantitative real-time-polymerase chain reaction (RT-PCR) indicating expression level alterations of pro- and antiapoptotic factors (e.g. BAX, BCL-2, survivin).

As long as a fluorescent microscopy system is available, applying microscopy techniques may represent a promising alternative to reveal apoptosis-specific changes in nuclear morphology. Here, we established a nuclear morphology assay for detection and quantification of nuclear morphology changes in apoptotic cells by fluorescence microscopy and subsequent analysis of visualized nuclear fluorescent signals.

\section{Materials and Methods}

Cell culture. Human epithelial cell lines LNCaP (prostate cancer; Cell Lines Service, Eppelheim, Germany) and MDA-MB-231 (breast cancer; American Type Culture Collection, Manassas, VA, USA) were propagated in RPMI 1640 medium supplemented with $10 \%$ fetal calf serum and 100 units/ml penicillin/streptomycin (PAN Biotech, Aidenbach, Germany) and DMEM/F12 medium (Invitrogen, Karlsruhe, Germany) containing 10\% fetal bovine serum (FBS; Sigma-Aldrich, Deisenhofen, Germany), $4.5 \mathrm{~g}$ glucose $/ 1,1 \mathrm{mM}$ sodium pyruvate and $50 \mu \mathrm{g} / \mathrm{ml}$ gentamycin (all from Invitrogen), respectively, at $37^{\circ} \mathrm{C}$ and $5 \% \mathrm{CO}_{2}$ in a humidified atmosphere. Cell sub-cultivation was realized twice a week by detachment of adherent cells applying $0.1 \%$ trypsin $/ 0.04 \%$ ethylenediaminetetraacetic acid (EDTA; PAN Biotech) and resuspension in the corresponding medium. For apoptosis induction, cells were incubated in the presence of $3.0 \mu \mathrm{M}$ cycloheximide (CHX; Merck, Darmstadt, Germany) with equal volumes of ethanol serving as vehicle control.

Proliferation assay. Cellular proliferation was examined by utilizing a CASY Cell Counter and Analyzer Model TT (Roche Applied Science, Mannheim, Germany) with $150 \mu \mathrm{m}$ capillary. Cells were detached by trypsin/EDTA treatment, suspended in CASYton (Roche Applied Science) and $400 \mu \mathrm{l}$ cell suspension of a 1:100 dilution was analyzed in triplicates. Cell line-specific gate settings were used to discriminate between viable cells, dead cells and cellular debris (LNCaP: $7.20 \mu \mathrm{m} / 15.45 \mu \mathrm{m}$; MDA-MB-231: $6.9 \mu \mathrm{M} / 12.0 \mu \mathrm{M}$ ).

TUNEL assay. Thirty thousand LNCaP cells and 5x104 MDA-MB231 cells were seeded into a 96-well cell culture plate and incubated for $24 \mathrm{~h}$ in the presence of $3 \mu \mathrm{M}$ CHX. TUNEL apoptosis analysis was performed using the HT TiterTACS Assay Kit (Trevigen, Gaithersburg, MD, USA) following the supplier's recommendations. Data were acquired using an Infinite 200 PRO multimode reader (Tecan, Männedorf, Switzerland) with i-Control 1.9 software (Tecan).

Nuclear morphology assay. For fluorescence microscope detection of nuclear morphology changes cells were incubated for $24 \mathrm{~h}$, washed twice with phosphate buffered saline (PBS; PAN Biotech) and permeabilized with $0.2 \%$ Triton X-100 (Carl Roth, Karlsruhe, Germany). For nucleus staining, cells were washed with PBS and incubated with $1.0 \mu \mathrm{g} / \mathrm{ml}$ 4',6-diamidino-2-phenylindole (DAPI; Carl Roth). Fluorescent microscopy images were obtained using a
BZ-9000 fluorescent microscope system (Keyence, Osaka, Japan) with a 20-fold magnification and the BZ II Analyzer software (Keyence). Morphological changes were determined by taking 10 images from different sites of a $3.5-\mathrm{cm}$ cell culture dish, which altogether represents $0.5 \%$ of the total growth area. The morphometric parameters were performed for each single nucleus of an image and calculated by the BZ II Analyzer software. Therefore, single nuclei were defined by the co-localization function of the Hybrid Cell Count BZ-H2C module as DAPI signal positive areas ranging from 1.0 to $200 \mu \mathrm{m}^{2}$. By defining these size criteria, small nuclear fragments, as well as nuclei clusters, were excluded from the analysis. Nuclear morphology parameters, as assessed in this study, were obtained by the BZ II Analyzer tools area (given in $\mu \mathrm{m}^{2}$ ), perimeter, major and minor axis (all given in $\mu \mathrm{m}$ ), as well as brightness (given in relative fluorescence units per cell).

Statistics. Data evaluation was performed using the Graph Pad Prism V 5.01 software (GraphPad Software, La Jolla, CA, USA). Statistical comparisons were made using the unpaired Student's $t$ test. Results of $p \leq 0.05(*), p \leq 0.01\left(^{* *}\right)$ and $p \leq 0.001$ (***) were considered significant. Data are given as mean \pm SD.

\section{Results}

CHX, a potent activator of apoptosis in several cell types, including epithelial cells of prostate and breast cancer (6-8), interferes with eukaryotic protein synthesis. When compared to control cells incubated with equal volumes of ethanol, administration of $3.0 \mu \mathrm{M}$ CHX led to significantly reduced proliferation rates of LNCaP cells $(24 \mathrm{~h}: 41.6 \pm 9.5 \%$, $p=0.0223 ; 48$ h: $19.4 \pm 1.7 \%, p=0.0442 ; 72$ h: $11.5 \pm 1.4 \%$, $p=0.0339$; Figure 1a), as well as of MDA-MB-231 cells (24 h: $60.9 \pm 2.1 \%, p=0.0002 ; 48$ h: $36.2 \pm 4.7 \%, p=0.0124 ; 72$ h: $18.2 \pm 1.5 \%, p=0.0001$; Figure $1 \mathrm{~b}$ ) within $72 \mathrm{~h}$ of treatment. The anti-proliferative effect of CHX was accompanied by the induction of apoptosis that was verified by TUNEL assay. In the presence of CHX, formation of chromosomal DNA fragments was clearly increased in both cell lines. TUNEL signals rose to $219.1 \pm 73.2 \%$ ( $p=0.0479, \mathrm{LNCaP}$; Figure 1c) and $152.6 \pm 27.7 \%(p=0.0479$, MDA-MB-231; Figure 1d) compared to vehicle-treated control cells.

According to growth kinetic studies, as shown in Figure 1a and b, fluorescence microscopy analysis of DAPI-stained cells caused reduced nuclei numbers in both cell lines after treatment with CHX (Figure 2a). Furthermore, size and shape of nuclei were apparently different in CHX and control samples. Software-based nucleus shape and nucleus staining intensity analysis indicated significant changes in nuclear morphology. Ten different fields of vision, equivalent to $0.5 \%$ of the total growth area, were analyzed encompassing 400 to 500 nuclei of CHX-treated cells and 700 to 1,000 nuclei of corresponding control cells. In the presence of CHX, nucleus size sank significantly, as demonstrated by diminished nuclear morphology parameters, such as area (LNCaP: $89.1 \pm 30.6 \%, p \leq 0.0001$, Figure 2b; MDA-MB-231: 91.6 \pm 28.0 , 

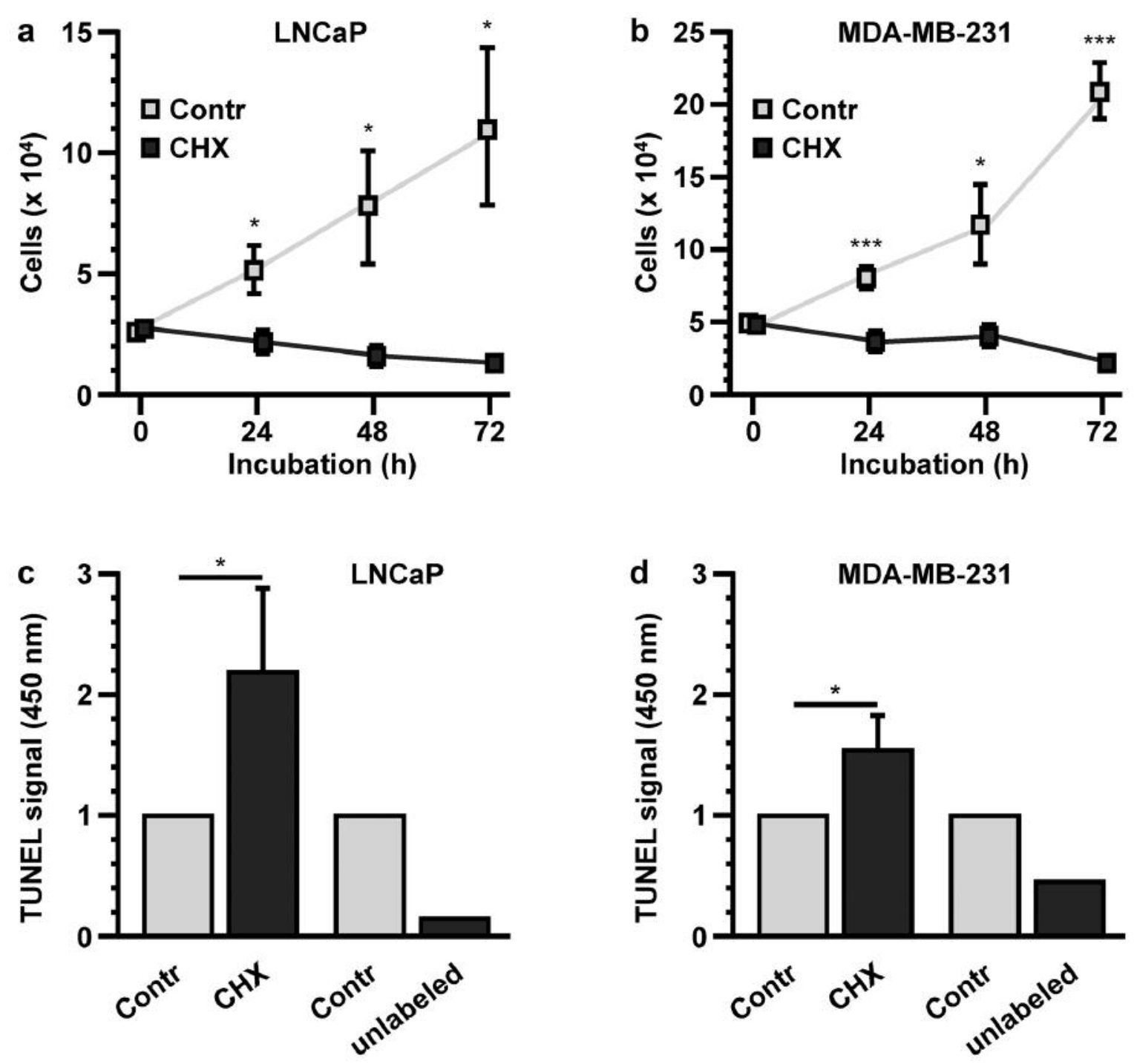

Figure 1. Apoptotic efficacy of cycloheximide (CHX) in an in vitro LNCaP and MDA-MB-231 cell model system. a, b: Growth inhibitory effect of $3.0 \mu M$ CHX in LNCaP cells (a) and MDA-MB-231 cells (b) incubated over a period of $72 \mathrm{~h}$. Data are mean \pm SD of viable cell count. $p \leq 0.05$ (*); $p \leq 0.01$ (**) and $p \leq 0.001$ (***) as determined by the Student's t-test. $c, d$ : Chromatin degradation detected by terminal deoxynucleotidyl transferasedUTP nick-end labeling (TUNEL) assay. Relative chromatin degradation in LNCaP cells (c) and MDA-MB-231 cells (d) after 24 h of incubation in the presence of CHX compared to vehicle treated controls. Data are given as the mean $\pm S D$ of relative TUNEL signal per cells with control-treated cells defined as $1.0 * p \leq 0.05 ; * * p \leq 0.01$ and $* * * p \leq 0.001$ as determined by the Student's $t$-test.

$p \leq 0.0001$, Figure $2 \mathrm{~g}$ ), perimeter (LNCaP: $95.6 \pm 19.4 \%$, $p=0.0001$, Figure 2c; MDA-MB-231: 96.3 $\pm 16.6 \%, p=0.0001$, Figure 2h), major axis (LNCaP: $95.7 \pm 20.3 \%, p=0.0002$, Figure 2d; MDA-MB-231: 97.6 $\pm 18.5 \%, p=0.0189$, Figure 2i) and minor axis (LNCaP: $93.3 \pm 20.1 \%, p \leq 0.0001$, Figure $2 \mathrm{e}$; MDA-MB-231: $93.5 \pm 16.2 \%, p \leq 0.0001$, Figure $2 \mathrm{j}$ ) compared to controls. Meanwhile, chromosomal DNA condensation was detectable and shown by elevated values of nuclear staining intensity (LNCaP: $102.0 \pm 13.4 \%, p=0.0388$, Figure 2f; MDAMB-231: $103.3 \pm 18.6 \%, p=0.0025$, Figure $2 \mathrm{k}$ ) compared to vehicle-treated control cells.

\section{Discussion}

Apoptosis measurement is frequently performed by fluorescence microscopy showing exemplary images of apoptotic bodies and chromatin condensation compared to non-apoptotic control cells (9-11). Although the underlying cellular processes are characteristic for apoptosis, the methodological evidence is limited by lack of quantification and statistical analysis of a sufficient number of nuclei.

In biological systems, a variety of microscopy approaches has been explored for the characterization of technical 

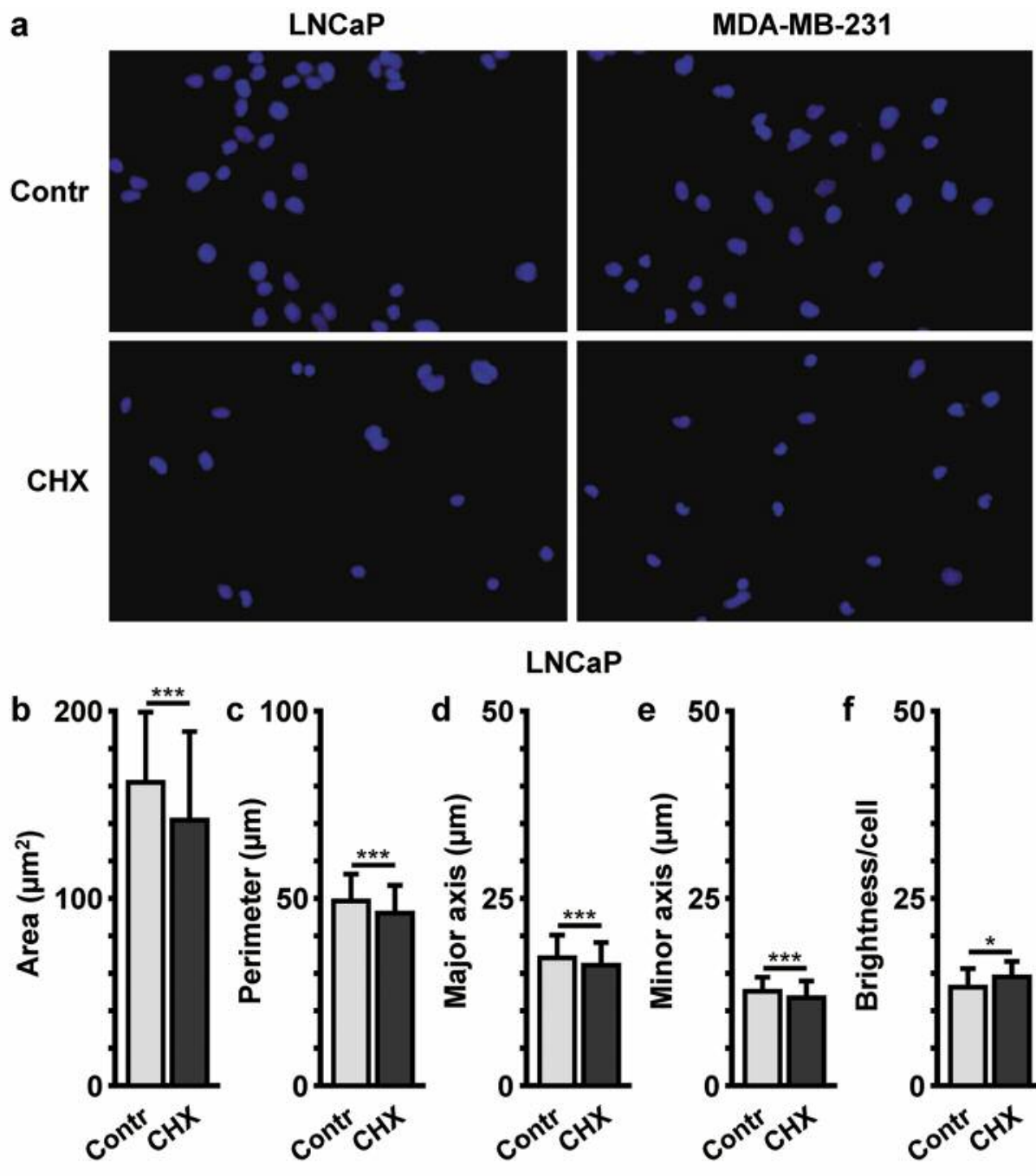

LNCaP
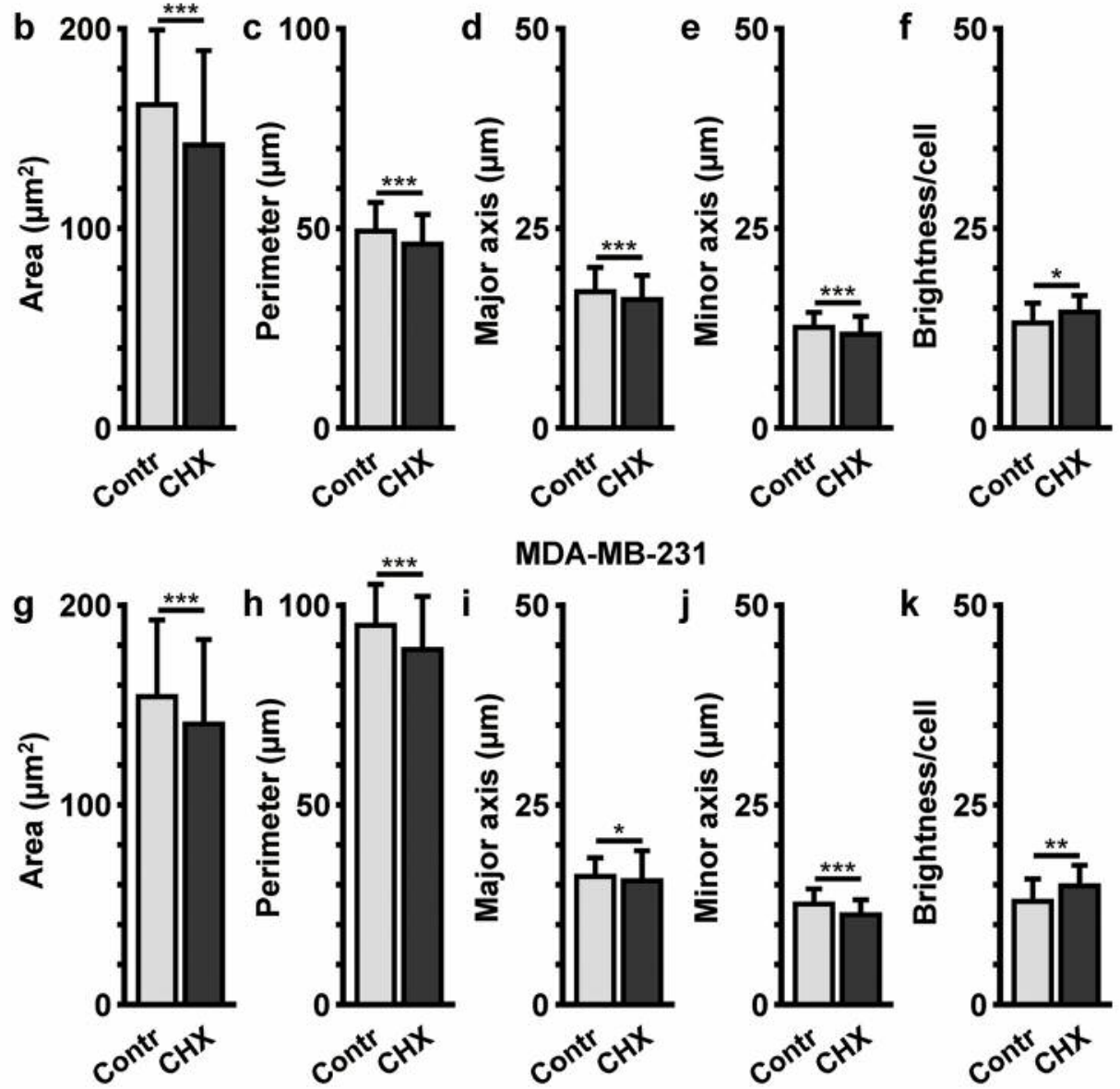

MDA-MB-231
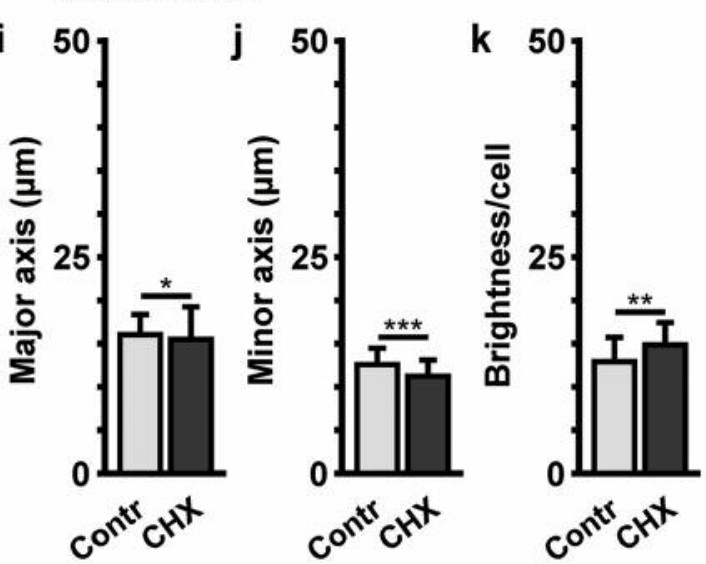

Figure 2. Nuclear morphology changes of 4',6-diamidino-2-phenylindole (DAPI)-stained LNCaP and MDA-MB-231 cells assessed by fluorescence microscopy. a: DAPI staining of LNCaP cells (left column) and MDA-MB-231 cells (right column) after 24 h of incubation in the presence of vehicle (control; upper row) and cycloheximide (CHX; lower row) using a BZ-9000 fluorescent microscope system with 20-fold magnification. b to k: Calculation of nuclear morphology parameters like area (LNCaP: b; MDA-MB-231: g), perimeter (LNCaP: c; MDA-MB-231: h), major axis (LNCaP: d; MDA-MB-231: i), minor axis (LNCaP: e; MDA-MB-231: j) and brightness (LNCaP: $f ;$ MDA-MB-231: k) by BZ II Analyzer software. Data are mean $\pm S D$ of calculated values. ${ }^{*} p \leq 0.05 ; * * \leq 0.01$ and $* * * p \leq 0.001$ as determined by the Student's $t$-test. 
materials like nanoparticles $(12,13)$. In the meantime, some studies have been performed using Image Pro Plus software (Media Cybernetics, Rockville, MD, USA) and ImageJ software (National Institute of Health, Bethesda, MD, USA) for analysis of stained nuclei by calculation of nuclear parameters $(14,15)$. Due to its high specificity and availability for quantification, DAPI has, thereby, become the method of choice in nucleus staining (16).

By utilizing a BZ-9000 fluorescent microscope system with BZ II Analyzer software, the nucleus size of LNCaP and MDA-MB-231 cancer cells was determined with mean nuclear area values of $\sim 150 \mu \mathrm{m}^{2}$, which is in accordance with literature data indicating that the eukaryotic nucleus size primarily varies in the range from 50 to $150 \mu \mathrm{m}^{2}(17,18)$. Using a computerized approach for the determination of nuclear parameters (area, perimeter, major and minor axis, brightness of nuclear DAPI signal), which were additionally confirmed by TUNEL assay, this study demonstrates capability of fluorescence microscopy to significantly detect apoptosis-specific modulations of nuclear morphology and, thus, provides an alternative tool for experimental apoptosis quantification in cell culture models. Additionally, these data suggest that it may be possible to successfully detect apoptotic nuclei in tissue samples; however, this must be evaluated in further studies. Moreover, and provided that the fluorescent microscopy device allows for automation, the automated and computerized signal detection and subsequent analysis appear feasible as routine method in laboratories.

The nuclear morphology assay described here might substitute the time-consuming, mostly kit-based and, thus, expensive apoptosis detection protocols of commercially available assays. The advantages are clearly evident: First, rested upon the principle of nuclear DNA staining with DNA intercalating fluorescent dyes (e.g. propidium iodide, DAPI), the nuclear morphology assay provides a simple and lowcost method for apoptosis detection. Second, analyzing nuclear morphology changes displays apoptotic events of several successive stages of apoptosis. While apoptosis assays based on molecular events are restricted to one apoptotic stage, nuclear morphology changes comprise early stages (e.g. chromatin condensation), as well as later and very late stages of apoptosis (e.g. nucleus shrinking and later on formation of apoptotic bodies). Consequently, apoptosis verification by nuclear morphology changes is more powerful than apoptosis assays based on one singular activity in the complex machinery of this cellular process.

Apoptotic bodies are small-sized vesicles of $<1.0 \mu \mathrm{m}$ carrying fragmented chromosomal DNA (19), thus appearing positive after DAPI staining, and absent in non-apoptotic cells. Since the resolution of commercially available fluorescent microscopy systems enables the detection of apoptotic bodies, we raised the question of whether this type of apoptotic marker may also be suitable for detection of apoptotic events. However, our observations, confirmed by a study from Diaz-Zamboni et al. (20), demonstrated complexity of apoptotic body detection relating to preparation artifacts and the need for specific image processing algorithms.

In conclusion, we presented a computerized method for apoptosis detection and quantification using images of fluorescent dye stained cell nuclei. The advantages of this nuclear morphology assay include the ability to routinely assess apoptosis by a fast, highly reproducible low-cost technique, the applicability of an experimental approach analyzing high numbers of single nuclei and the detection of apoptosis in early, as well as late, stages of the apoptotic cascade.

\section{Acknowledgements}

The Authors thank Anne Brandenburg and Katja Wittig for excellent technical assistance.

\section{References}

1 Salomons GS, Smets LA, Verwijs-Janssen M, Hart AA, Haarman EG, Kaspers GJ, Wering EV, Der Does-Van Den Berg AV and Kamps WA: Bcl-2 family members in childhood acute lymphoblastic leukemia: Relationships with features at presentation, in vitro and in vivo drug response and long-term clinical outcome. Leukemia 13: 1574-1580, 1999.

2 Weiss M, Gümbel D, Hanschmann E, Mandelkow R, Gelbrich N, Zimmermann U, Walther R, Ekkernkamp A, Sckell A, Kramer A, Burchardt M, Lillig CH and Stope MB: Cold atmospheric plasma treatment induces anti-proliferative effects in prostate cancer cells by redox and apoptotic signaling pathways. PLoS ONE 10: e0130350, 2015.

3 Grossebrummel H, Peter T, Mandelkow R, Weiss M, Muzzio D, Zimmermann U, Walther R, Jensen F, Knabbe C, Zygmunt M, Burchardt M and Stope MB: Cytochrome P450 17A1 inhibitor abiraterone attenuates cellular growth of prostate cancer cells independently from androgen receptor signaling by modulation of oncogenic and apoptotic pathways. Int J Oncol 48: 793-800, 2016.

4 Kerr JF, Wyllie AH and Currie AR: Apoptosis: a basic biological phenomenon with wide-ranging implications in tissue kinetics. Br J Cancer 26: 239-257, 1972.

5 Majno G and Joris I: Apoptosis, oncosis, and necrosis. An overview of cell death. Am J Pathol 146: 3-15, 1995.

6 Coffey RN, Watson RW, Hegarty PK, Watson CL, Wolohan L, Brady HR, O'Keane C and Fitzpatrick JM: Priming prostate carcinoma cells for increased apoptosis is associated with upregulation of the caspases. Cancer 92: 2297-2308, 2001.

7 Alessenko AV, Boikov P, Filippova GN, Khrenov AV, Loginov AS and Makarieva ED: Mechanisms of cycloheximide-induced apoptosis in liver cells. FEBS Lett 416: 113-116, 1997.

8 Tan Y, Teng E and Ting AE: A small inhibitor of the interaction between $\mathrm{Bax}$ and $\mathrm{Bcl}-\mathrm{X}(\mathrm{L})$ can synergize with methylprednisolone to induce apoptosis in $\mathrm{Bcl}-\mathrm{X}(\mathrm{L})$-overexpressing breast-cancer cells. J Cancer Res Clin Oncol 129: 437-448, 2003. 
9 Saha SK, Sikdar S, Mukherjee A, Bhadra K, Boujedaini N and Khuda-Bukhsh AR: Ethanolic extract of the Goldenseal, Hydrastis canadensis, has demonstrable chemopreventive effects on HeLa cells in vitro: Drug-DNA interaction with calf thymus DNA as target. Environ Toxicol Pharmacol 36: 202-214, 2013.

10 Ansil PN, Wills PJ, Varun R and Latha MS: Cytotoxic and apoptotic activities of Amorphophallus campanulatus (Roxb.) B1. tuber extracts against human colon carcinoma cell line HCT- 15 . Saudi J Biol Sci 21: 524-531, 2014.

11 Peng C, Huang C, Hsu S and Wang C: Penta-acetyl geniposide induce apoptosis in $\mathrm{C} 6$ glioma cells by modulating the activation of neutral sphingomyelinase-induced $\mathrm{p} 75$ nerve growth factor receptor and protein kinase Cdelta pathway. Mol Pharmacol 70: 997-1004, 2006.

12 Wissing SA and Muller RH: Solid lipid nanoparticles (SLN) - a novel carrier for UV blockers. Pharmazie 56: 783-786, 2001.

13 Cioran AM, Musteti AD, Teixidor F, Krpetic Z, Prior IA, He Q, Kiely CJ, Brust M and Vinas C: Mercaptocarborane-capped gold nanoparticles: Electron pools and ion traps with switchable hydrophilicity. J Am Chem Soc 134: 212-221, 2012.

14 Eidet JR, Pasovic L, Maria R, Jackson CJ and Utheim TP: Objective assessment of changes in nuclear morphology and cell distribution following induction of apoptosis. Diagn Pathol 9: 92, 2014.

15 Lipiec EW, Wiechec A, Dulinska-Litewka J, Kubica M, Lekki J, Stachura Z, Wiltowska-Zuber J and Kwiatek WM: Changes in cellular response to the damage induced in $\mathrm{PC}-3$ prostate cancer cells by proton microbeam irradiation. Gen Physiol Biophys 31 : 11-18, 2012.
16 Tarnowski BI, Spinale FG and Nicholson JH: DAPI as a useful stain for nuclear quantitation. Biotech Histochem 66: 297-302, 1991.

17 Kazanowska B, Jelen M, Reich A, Tarnawski W and Chybicka A: The role of nuclear morphometry in prediction of prognosis for rhabdomyosarcoma in children. Histopathology 45: 352-359, 2004.

18 Czekaj P, Palasz A, Lebda-Wyborny T, Nowaczyk-Dura G, Karczewska W, Florek E and Kaminski M: Morphological changes in lungs, placenta, liver and kidneys of pregnant rats exposed to cigarette smoke. Int Arch Occup Environ Health 75 Suppl: S27-S35, 2002.

19 Agouni A, Andriantsitohaina R and Martinez MC: Microparticles as biomarkers of vascular dysfunction in metabolic syndrome and its individual components. Curr Vasc Pharmacol 12: 483-492, 2014.

20 Diaz-Zamboni JE, Adur JF, Vicente N, Fiorucci MP, Izaguirre MF and Casco VH: 3D automatic quantification applied to optically sectioned images to improve microscopy analysis. Eur J Histochem 52: 115-126, 2008. 